Session: Manipulating Fat for Plant Based Product Development

\title{
Effect of High-intensity Ultrasound on the Oleogelation and Physical Properties of High Melting Point Monoglycerides and Triglycerides Oleogels
}

Thursday, May 6, 2021 (J) 5:50 PM - 6:10 PM CDT

Presenting Author(s)

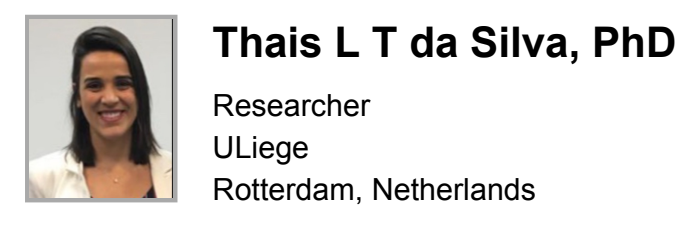

\section{Co-Author(s)}

\begin{tabular}{l|l} 
& Sabine Danthine, PhD \\
SD & $\begin{array}{l}\text { Professor } \\
\text { University of Liège } \\
\text { Gembloux, Namur, Belgium }\end{array}$ \\
\hline
\end{tabular}

\begin{abstract}
Oleogels and oleogelation routes have been extensively studied in the past decade; however the industry has not yet implemented this technique due to price, availability and clean label. The objective of this study was to evaluate the synergism of binary oleogels structured by monoglycerides (MG) and high melting point triacylglycerols (HF) with and without high-intensity ultrasound (HIU) according to their physical properties. MG:HF (0:6, 1:5, 2:4, 3:3, 4:2, 5:1 and 6:0) oleogels were produced by mixing at $70^{\circ} \mathrm{C}$ with a stirring of $350 \mathrm{rpm}$ for $5 \mathrm{~min}$, followed by a cooling and storage at $20^{\circ} \mathrm{C}$ for $24 \mathrm{~h}$. A $20 \mathrm{kHz}$ HIU was applied for $10 \mathrm{~s}, 30 \mathrm{~s}$ or $10 \mathrm{~s}$ using 3 pulses (10sON/10sOFF) during the cooling step via macro tip $(12.7 \mathrm{~mm})$ and $50 \%$ amplitude $(56 \mathrm{~W})$ in the presence of few crystals. Samples were evaluated according to their hardness, oil binding capacity $(\mathrm{OBC})$, microstructure, melting behavior, viscoelasticity and flow behavior. The best physical properties were found in the MG6:HF0 oleogel, with a hardness of $1.2 \mathrm{~N}$, elasticity of $5.5 \mathrm{kPa}$, viscosity of $99 \mathrm{~Pa}$.s and $99 \% \mathrm{OBC}$. These properties were reduced with the decrease of $\mathrm{MG}$ in the blend. The
\end{abstract}


sonication did not improve the MG6:HF0, instead it affected its properties negatively. However, sonication showed a positive effect on the blends of MG and HF. The hardness was improved at least 3 -fold and OBC around $20 \%$, these effects were already observed using only 10 s sonication.

Sonocrystallization induced secondary nucleation and changed the crystalline material only in blends containing HF indicating the better effect of the sonocrystallization on oleogels in the presence of high-melting points triacylglycerols.

Description: Oleogels are a valuable alternative in food industry to replace trans and reduce saturated fatty acids content in many food products. The combination of a binary structuration and use of high-intensity ultrasound that is a physical green technology will give the food industry information on how to improve the physical properties of oleogels without increasing the amount of oleogelators, giving a future alternative to clean label and sensory claims of oleogels applications. 